\title{
Pixel value analysis for detection of simulated early external root resorption
}

\section{Marcelo Lupion POLETI (a) \\ Thais Maria Freire FERNANDES(b) \\ Camila Cristina PAIZ ${ }^{(\mathrm{c})}$ \\ Izabel Regina Fischer RUBIRA- \\ BULLEN(d) \\ Ana Lúcia Alvares CAPELOZZA ${ }^{\text {(d) }}$}

(a) Department of Oral Radiology, Faculty of Dentistry, Universidade Norte do Paraná UNOPAR, Londrina, PR, Brazil.

(b) Department of Orthodontics, Faculty of Dentistry, Universidade Norte do Paraná UNOPAR, Londrina, PR, Brazil.

(c) Private practice, Santa Rita do Passa Quatro, SP, Brazil.

(d) Department of Stomatology, Bauru School of Dentistry, Universidade de São Paulo USP, Bauru, SP, Brazil.

Declaration of Interests: The authors certify that they have no commercial or associative interest that represents a conflict of interest in connection with the manuscript.

\section{Corresponding Author:}

Marcelo Lupion Poleti

E-mail: marcelo_poleti@yahoo.com.br

DOI: 10.1590/1807-3107BOR-2014.vol28.0045

Submitted: Feb 10, 2014

Accepted for publication: Jun 01, 2014

Last revision: Aug 12, 2014
Abstract: The aim of this study was to determine the efficacy of pixel value analysis using images generated by the Digora ${ }^{\mathrm{TM}}$ and Visualix ${ }^{\mathrm{TM}}$ systems for the early detection of external root resorption (ERR). Thirty extracted human lower incisors were radiographed using the Digora and Visualix systems; then, ERR was induced by immersing the teeth in 6 mol L-1 of hydrochloric acid for different periods of time (10, 30 and 60 minutes). ERR was confirmed by calcium quantification with an atomic absorption spectrophotometer. One digital image was acquired per time period at $70 \mathrm{kVp}, 7 \mathrm{~mA}$, $2.2 \mathrm{~mm}$ filtration, focus-film distance of $30 \mathrm{~cm}$, and with exposure times of $0.09 \mathrm{~s}$ in the Digora system and $0.05 \mathrm{~s}$ in Visualix system. The region of interest was defined using ImageJ software. Statistical analysis was performed using ANOVA and Pearson's correlation $(p<0.05)$. There was no statistically significant difference between the time for ERR induction and the pixel values with either system. A positive correlation between the time of ERR induction and the calcium concentration was observed $(r=0.8892 ; p<0.001)$. In conclusion, independent of the site of ERR induction and the digital system, pixel value analysis was not effective for ERR detection.

Keywords: Radiography, Dental, Digital; Root Resorption; Early Diagnosis.

\section{Introduction}

Early and accurate detection of external root resorption (ERR) during orthodontic treatment is essential to identify teeth at risk of severe resorption. ${ }^{1,2}$ Early detection of ERR allows orthodontic treatment plans to be modified to minimize or prevent continued loss of root structure. ${ }^{1,2}$ Periapical radiographic imaging, either conventional or digital, is usually the method of choice for detection of apical $\mathrm{ERR}^{3,4,5}$ despite reports that cone beam computed tomography (CBCT) is more reliable than intraoral radiography. ${ }^{2,6}$ However, there are differences between the techniques. Conventional radiographic images are acquired by the exposure of silver crystals in an emulsion to light, followed by chemical processing. In the case of digital images, the sensor is triggered by the same rays as those used for conventional imaging, but the processing is performed by means of digital scanning. Because the sensors used in dental radiology exhibit high sensitivity to x-rays, lower doses of radiation than those used in the conventional technique may be used. In addition, digital scanning with 
a charge-coupled device (CCD), a complementary metal oxide semiconductor (CMOS) and a photostimulable phosphor plate (PSP) eliminates the errors incurred during the chemical processing phase of conventional radiography.

Digital images are generated by a combination of pixels that contain information about the color or grey levels at each pixel location. ${ }^{7}$ Another relevant issue concerns the limitations of human vision, wherein approximately 32 shades of grey can be distinguished, 7 8-bit digital images surpass the limits of human visual detection in terms of distinguishing among 256 shades of grey. ${ }^{8,9}$ Visual evaluation of resorption using radiographs has proven to be highly inaccurate because of magnification errors and inability to be readily repeated and reproduced. ${ }^{10,11}$ Thus, the radiographic acquisition of images with high grey-scale discrimination results in greater sensitivity to the loss or gain of mineralized structures and could detect early ERR.

Radiographic studies have reported conflicting results regarding the correlation between ERR and orthodontic treatment. The main reason for such variability in results might be a lack of standardization of the methods, which limits the comparability of the results obtained by different studies. ${ }^{12,13}$ Because of the disagreement among the results of non-standardized scientific experiments, the aim of the present study was to assess the use of a digital tool (analysis based on pixel values) for the detection of ERR using images acquired with two different digital systems.

\section{Methodology}

Thirty human lower incisors were obtained at the tooth bank of the Bauru School of Dentistry, Universidade de São Paulo - USP, according to protocols established by the institutional Research Ethics Committee. The teeth were stored in a saline solution at room temperature throughout the study. The teeth were initially subjected to scaling and planing of the roots using ultrasound to remove any calculus, as in most cases, the indication for exodontia had been periodontal disease.
This study was approved by the Research Ethics Committee of the Bauru School of Dentistry, USP (Protocol no. 50/2008).

The teeth were then randomly allocated to two groups ( $n=15)$ defined by the localization of the chemically induced lesions as follows: Group P - teeth with ERR induced on the proximal surface, and Group B - teeth with ERR induced on the buccal surface.

After drying the tooth, a $2 \mathrm{~mm} \times 2 \mathrm{~mm}$ wax sheet was placed on the proximal or buccal apical surface of the root to define the area to be subjected to acidinduced chemical resorption. Subsequently, the full surface of the root was coated with two thin layers of an acid-resistant material (nail polish) with 1-hour intervals between applications.

The wax was removed, and the root surface was exposed to $6 \mathrm{~mol} \mathrm{~L}^{-1}$ hydrochloric acid to induce resorption. ${ }^{14,15}$ Thirty teeth were subjected to this treatment. Induction was performed 24 hours after the application of the insulating material and consisted of the immersion of the teeth in acid for time periods of three different lengths $(10,30$, and 60 minutes). ${ }^{14}$ After each period of induction, the teeth were rinsed in tap water for 10 minutes to remove the acid and to interrupt the process of resorption; then, the teeth were radiographed. ERR was confirmed by calcium quantification of the acid solution using atomic absorption spectrophotometry using a colorimetric method (Thermo Scientific, Waltham, USA), after each step of the demineralization process. ${ }^{14}$

To analyze resorption using the pixel values, digital images of the teeth were acquired using the $\operatorname{Digora}^{\text {TM }}$ (Soredex, Orion Corporation, Helsinki, Finland) and Visualix ${ }^{\mathrm{TM}}$ eHD (Gendex, Dental Systems, Milan, Italy) systems.

To standardize the image acquisition, a device was constructed where the teeth were placed in the alveoli of a dry mandible coupled to an acrylic base to simulate the hard and soft tissues, respectively, and to hold the sensors parallel and close to the radiographed area. Next, the teeth were radiographed using a dental device (Yoshida Dental MFC Co. Ltd., Tokyo, Japan) at 70 kV, 7 mA, 2.2 $\mathrm{mm}$ filtration and with a focus-film distance of 30 $\mathrm{cm}$. The exposure times were defined in a pilot 
study as $0.05,0.07,0.09,0.1,0.13,0.17$, and $0.2 \mathrm{~s}$. The images were assessed by two experienced radiologists who were blinded to the exposure times. The images from each digital system that exhibited the best sharpness, density, and contrast were rated as adequate. Therefore, according to the previously established selection criteria, the exposure times used in the present study were $0.09 \mathrm{~s}$ for the Digora $^{\mathrm{TM}}$ system and $0.05 \mathrm{~s}$ for the Visualix ${ }^{\mathrm{TM}}$ system.

The Digora ${ }^{\mathrm{TM}}$ is a PSP system that comprises reusable (white or blue) phosphor plates and a scanning system coupled to a personal computer. In this study, a blue phosphor plate was used, which was turned off immediately before each test to avoid any residual signal caused by the ambient light during storage. The system was calibrated for the exposures to be performed.

The Visualix ${ }^{\mathrm{TM}}$ eHD system comprises an eHD sensor, which is a charge-coupled device (CCD). The CCD is coated with a layer of cesium iodide, which is a luminescent material that increases the interaction between the sensor and $x$-rays, and a layer of amorphous carbon. In this system, the sensor is directly connected to a personal computer by a cable. The images acquired by means of the phosphor plate or the eHD sensor were processed by the software included in each system and were stored in TIFF format (without compression) to permit later processing with other software.

After all of the radiographic images were acquired, a 17-inch flat-panel screen computer (A505-S6975, Satellite, Toshiba, Tokyo, Japan) was used to assess them. The control image for each system (Digora ${ }^{\mathrm{TM}}$ and Visualix ${ }^{\mathrm{TM}}$ ) was opened and inverted using ImageJ software (National Institutes of Health, Bethesda, USA) to facilitate the distinction between the external surface of the root and the alveolar bone. Next, the region of interest (ROI) was defined manually to represent the external apical surface of the root and was saved in a file for the size ( $3 \mathrm{~mm}$ height) and format to be applied to all of the measurements performed in each tooth (Figure 1).

After the ROIs were defined, the software supplied data (histograms) on the average pixel values on an 8-bit scale, where 0 corresponded to the dark-

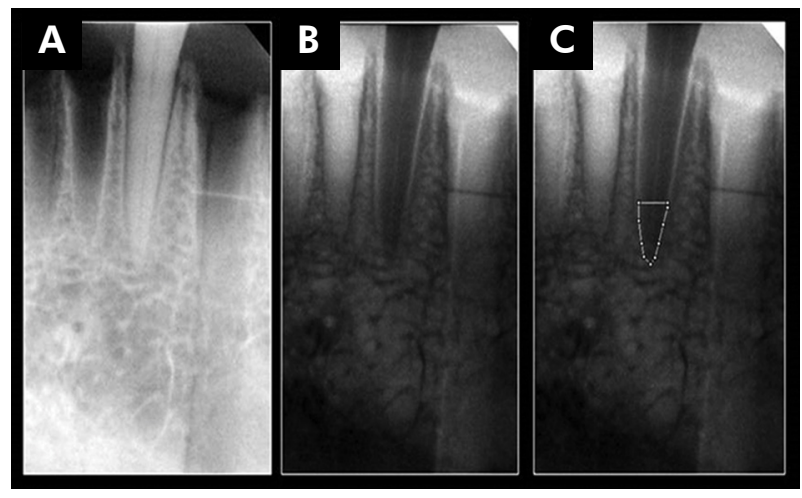

Figure 1. A) Original image, B) inverted image and C) the region of interest (ROI) defined manually using ImageJ software.

est grey shade (black) and 255 corresponded to the lightest grey shade (white). No image processing technique was used to enhance the images.

Analysis of the pixel values was performed by an examiner before (control) and after the induction of ERR (10, 30, and 60 minutes) in each tooth of Groups $\mathrm{P}$ and $\mathrm{B}$ with both image acquisition systems.

To analyze the correlation between the amount of calcium removed during the ERR process and the pixel values, a Pearson's correlation test of the data was performed. Analysis of variance (ANOVA) was used to assess the differences between the average pixel values of the root resorption area in groups $\mathrm{P}$ and $\mathrm{B}$ for each system (Digora ${ }^{\mathrm{TM}}$ and Visualix ${ }^{\mathrm{TM}}$ ). Intraclass correlation coefficients (ICC) and the BlandAltman test were used to determine intraobserver reliability. Statistical analysis was performed using Statistica for Windows 6.0 software (Statistica for Windows, StatSoft, Inc. Tulsa, USA), and the significance level was established as $p<0.05$.

\section{Results}

Intraobserver reliability for both the Digora (1.00) and Visualix (0.97) systems was determined to be very good using ICC and the Bland-Altman test. These results reflect high intraobserver reliabilities.

Table 1 shows the comparison between the total calcium removed and the time of induced ERR for proximal and buccal lesions. There was a positive correlation $(r=0.8892 ; p<0.001)$ between the time of ERR induction and the resulting amount of calcium removed. 
Table 1. Comparison between the total calcium removed and time of induced ERR for the proximal and buccal regions.

\begin{tabular}{lcc}
\hline \multirow{2}{*}{ Time of induced ERR $(\mathrm{min})$} & \multicolumn{2}{c}{ Total Calcium removed $(\mathrm{mg} / \mathrm{ml})$} \\
\cline { 2 - 3 } & Proximal & Buccal \\
\hline 0 & 0.00 & 0.00 \\
10 & 6.54 & 1.89 \\
30 & 9.61 & 3.91 \\
60 & 14.55 & 22.30 \\
\hline
\end{tabular}

The means, standard deviations, and analysis of variance for the pixel values of the proximal and buccal lesions are presented in Table 2. No statistically significant differences were found between the investigated systems (Digora ${ }^{\mathrm{TM}}$ and Visualix ${ }^{\mathrm{TM}}$ ) at any of the times of ERR induction. tometry, ${ }^{14}$ and the teeth subjected to the longest ERR induction time exhibited the greatest calcium release (Table 1 and Pearson's correlation). Therefore, this method of inducing root resorption is practical, simulates the clinical conditions of ERR, and might be applied in similar studies.

The diagnosis of ERR in clinical situations is usually based on conventional and digital radiographic images. ${ }^{3,4,5}$ In the present study, ERR was assessed by means of the pixel values on images acquired using two types of digital radiographic systems, PSP and CCD.

The advent of digital imaging allowed simplified post-processing and transfer of radiographic information because the images acquired by the

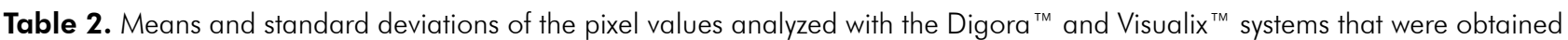
at the different times of ERR induction (ANOVA).

\begin{tabular}{|c|c|c|c|c|c|c|c|c|c|}
\hline & \multicolumn{9}{|c|}{ Pixel values } \\
\hline & \multicolumn{2}{|c|}{ TO } & \multicolumn{2}{|c|}{$\mathrm{T} 10$} & \multicolumn{2}{|c|}{ T30 } & \multicolumn{2}{|c|}{ T60 } & \multirow[t]{2}{*}{$p$-value } \\
\hline & Mean & SD & Mean & SD & Mean & SD & Mean & SD & \\
\hline \multicolumn{10}{|l|}{ Digora } \\
\hline Proximal & 178.52 & 36.11 & 194.22 & 22.98 & 202.67 & 16.76 & 181.96 & 29.60 & 0.155 \\
\hline Buccal & 152.49 & 21.32 & 150.85 & 21.08 & 136.48 & 30.59 & 146.69 & 25.55 & 0.620 \\
\hline \multicolumn{10}{|l|}{ Visualix } \\
\hline Proximal & 204.57 & 3.30 & 203.45 & 4.00 & 203.96 & 3.45 & 204.38 & 3.34 & 0.912 \\
\hline Buccal & 207.44 & 4.79 & 210.91 & 8.10 & 210.77 & 7.19 & 205.47 & 6.08 & 0.260 \\
\hline
\end{tabular}

\section{Discussion}

The detection of small ERR lesions during orthodontic treatment is essential to identify teeth at risk for severe resorption, and the timely ability to quantify the lesions associated with ERR is valuable. ${ }^{1}$ Some in vitro and ex vivo studies simulating ERR lesions with drills have been performed. ${ }^{2,3,4,5,6,16,17,18}$ However, the margins of the lesions artificially produced with drills in these studies are sharper than those of natural lesions, whose margins tend to be irregular. In the present study, simulated ERR was induced chemically because this procedure affords ill-defined margins that are similar to those with ERR of biological origin and clinical condition. ${ }^{14,15}$ In addition, ERR was confirmed by quantification of the calcium removed using atomic absorption spectropho- electronic sensor are stored in the computer and can be handled using the appropriate software. ${ }^{19,20}$ Such software might improve visualization and quantification of the images because it permits the adjustment of brightness and contrast, rotation of the images and linear and angular measurements. ${ }^{21}$ The goal of post-processing is to efficiently extract diagnostically useful information and to discard unimportant information, but high-quality radiographs are crucial for accurate diagnosis, ${ }^{20,22}$ therefore, the analysis of pixel values was included in the present study for the quantitative analysis of ERR on digital radiographic images. ImageJ software was chosen to quantify the pixel values because it is open-source, it is updated on a regular basis by a reliable source, and its plug-in tools are user-friendly. 
The pilot test was of paramount importance for analysis of the radiographs because it established the most appropriate exposure time for each digital system. ${ }^{3}$ When digital images are generated without taking into account the time of exposure needed for individual digital systems, significant noise may be added to the images, which particularly interferes with pixel-density analysis., ${ }^{7,23,24}$

In this study, we used acrylic and a dry mandible to simulate the soft and hard tissues, respectively, to mimic natural conditions and to standardize the acquisition of radiographic images. ${ }^{25}$ According to Pietrobelli et al., ${ }^{26}$ an attenuation phenomenon occurs when photons pass through certain elements, chemical components, or solutions, whereby their intensity decreases spontaneously due to atomic interactions, resulting in the absorption and dissipation of such photons. Consequently, when $\mathrm{x}$-ray beams are directed towards a tooth, the soft and hard tissues interposed in their trajectory influence the resulting pixel values.

When the ERR induction time and the pixel values were assessed in the Digora ${ }^{\mathrm{TM}}$ and Visualix ${ }^{\mathrm{TM}}$ systems, we expected that increases in ERR induction time would reduce the pixel values. However, no significant differences were observed for either digital system in either the proximal or the buccal region (Table 2). This result may have occurred due to a lack of reproducibility and/or to noise generated by these systems. Better results may have been obtained if the immersion times in the acid had been

\section{References}

1. Levander E, Malmgren O. Evaluation of the risk of root resorption during orthodontic treatment: a study of upper incisors. Eur J Orthod. 1988 Feb;10(1):30-8.

2. Ponder SN, Benavides E, Kapila S, Hatch NE. Quantification of external root resorption by low- vs high-resolution conebeam computed tomography and periapical radiography: a volumetric and linear analysis. Am J Orthod Dentofacial Orthop. 2013 Jan;143(1):77-91.

3. Kamburoglu K, Tsesis I, Kfir A, Kaffe I. Diagnosis of artificially induced external root resorption using conventional intraoral film radiography, CCD, and PSP: an ex vivo study. Oral Surg Oral Med Oral Pathol Oral Radiol Endod. 2008 Dec;106(6):885-91. longer; however, the aim of this study was to evaluate the ability of the systems to detect early ERR.

Freitas et al..$^{27}$ tested the reproducibility of the pixel values generated by the Digora ${ }^{\mathrm{TM}}$ system. Their exposures were standardized with variations of the time of exposure. These authors concluded that the reproducibility of such data with the Digora ${ }^{\mathrm{TM}}$ system was unsatisfactory and that their findings could explain unexpected variations in pixel values, such as the variability that occurred in the present study. Therefore, studies of bone gain or loss using pixel values might result in false positive results because the variability detected in such alterations might be due to the system used and not due to the lesions themselves.

Noise may be defined as an undesirable fluctuation of pixel intensity that affects the sensitivity of an image. ${ }^{7,23,24}$ Noise varies considerably as a function of the quality of the system used and the time of exposure, ${ }^{7}$ and it can arise from the processing of electronic signals or be determined by the quantity of photons. ${ }^{23,28}$ In the present study, there was greater noise in the Digora ${ }^{\mathrm{TM}}$ system as indicated by the large standard deviations.

\section{Conclusions}

Based on the methods used, the results suggest that independent of the site of ERR induction and the digital system used, pixel value analysis was not effective for ERR detection.

4. Ono E, Medici Filho E, Faig Leite H, Tanaka JL, De Moraes ME, De Melo Castilho JC. Evaluation of simulated external root resorptions with digital radiography and digital subtraction radiography. Am J Orthod Dentofacial Orthop. 2011 Mar;139(3):324-33.

5. Westphalen VP, Gomes de Moraes I, Westphalen FH, Martins WD, Souza PH. Conventional and digital radiographic methods in the detection of simulated external root resorptions: a comparative study. Dentomaxillofac Radiol. 2004 Jul;33(4):233-5.

6. Ren H, Chen J, Deng F, Zheng L, Liu X, Dong Y. Comparison of cone-beam computed tomography and periapical radiography for detecting simulated apical root resorption. Angle Orthod. 2013 Mar;83(2):189-95. 
7. Farman AG, Scarfe WC. Pixel perception and voxel vision: constructs for a new paradigm in maxillofacial imaging. Dentomaxillofac Radiol. 1994 Feb;23(1):5-9.

8. Velders XL, Sanderink GC, van der Stelt PF. Dose reduction of two digital sensor systems measuring file lengths. Oral Surg Oral Med Oral Pathol Oral Radiol Endod. 1996 May;81(5):607-12.

9. Wenzel A. Sensor noise in direct digital imaging (the RadioVisioGraphy, Sens-a-Ray, and Visualix/Vixa systems) evaluated by subtraction radiography. Oral Surg Oral Med Oral Pathol. 1994 Jan;77(1):70-4.

10. Brezniak N, Goren S, Zoizner R, Dinbar A, Arad A, Wasserstein $A$, et al. The use of an individual jig in measuring tooth length changes. Angle Orthod. 2004 Dec;74(6):780-5.

11. Chan EK, Darendeliler MA. Exploring the third dimension in root resorption. Orthod Craniofac Res. 2004 May;7(2):64-70.

12. Brezniak N, Wasserstein A. Root resorption after orthodontic treatment: Part 2. Literature review. Am J Orthod Dentofacial Orthop. 1993 Feb;103(2):138-46.

13. Reukers E, Sanderink G, Kuijpers-Jagtman AM, van't Hof M. Assessment of apical root resorption using digital reconstruction. Dentomaxillofac Radiol. 1998 Jan;27(1):25-9.

14. Eraso FE, Parks ET, Roberts WE, Hohlt WF, Ofner S. Density value means in the evaluation of external apical root resorption: an in vitro study for early detection in orthodontic case simulations. Dentomaxillofac Radiol. 2007 Mar;36(3):130-7.

15. Kravitz LH, Tyndall DA, Bagnell CP, Dove SB. Assessment of external root resorption using digital subtraction radiography. J Endod. 1992 Jun;18(6):275-84

16. Durack C, PatelS, Davies J, Wilson R, Mannocci F. Diagnostic accuracy of small volume cone beam computed tomography and intraoral periapical radiography for the detection of simulated external inflammatory root resorption. Int Endod J. 2011 Feb;44(2):136-47.

17. Hintze H, Wenzel A, Andreasen FM, Swerin I. Digital subtraction radiography for assessment of simulated root resorption cavities. Performance of conventional and reverse contrast modes. Endod Dent Traumatol. 1992 Aug;8(4):149-54.
18. Shokri A, Mortazavi H, Salemi F, Javadian A, Bakhtiari H, Matlabi H. Diagnosis of simulated external root resorption using conventional intraoral film radiography, CCD, PSP, and CBCT: a comparison study. Biom J. 2013 Jan-Feb;36(1):18-22.

19. Grondahl HG. Digital radiology in dental diagnosis: a critical view. Dentomaxillofac Radiol. 1992 Nov;21(4):198-202.

20. Versteeg CH, Sanderink GC, van der Stelt PF. Efficacy of digital intra-oral radiography in clinical dentistry. J Dent. 1997 May-Jul;25(3-4):215-24.

21. Nelvig P, Wing K, Welander U. Sens-A-Ray. A new system for direct digital intraoral radiography. Oral Surg Oral Med Oral Pathol. 1992 Dec;74(6):818-23.

22. Sanderink GC. Imaging: new versus traditional technological aids. Int Dent J. 1993 Aug;43(4):335-42.

23. Molteni R. Direct digital dental x-ray imaging with visualix/ VIXA. Oral Surg Oral Med Oral Pathol. 1993 Aug;76(2):235-43.

24. Rubira-Bullen IR, Escarpinati MC, Schiabel H, Vieira MA, Rubira $\mathrm{CM}$, Lauris JR. Evaluating noise in digitized radiographic images by means of histogram. J Appl Oral Sci. 2006 Dec;14(6):410-4.

25. Cook JE, Cunningham JL. The assessment of fracture healing using dual x-ray absorptiometry: a feasibility study using phantoms. Phys Med Biol. 1995 Jan;40(1):119-36.

26. Pietrobelli A, Formica C, Wang Z, Heymsfield SB. Dual-energy X-ray absorptiometry body composition model: review of physical concepts. Am J Physiol. 1996 Dec;271(6 Pt 1):E941-51.

27. Freitas P, Yaedu RY, Rubira-Bullen IR, Escarpinati M, Vieira MC, Schiabel H, et al. Reproducibility of pixel values for two photostimulable phosphor plates in consecutive standardized scannings. Braz Oral Res. 2006 Jul-Sep;20(3):207-13.

28. Borg E, Attaelmanan A, Grondahl HG. Subjective image quality of solid-state and photostimulable phosphor systems for digital intra-oral radiography. Dentomaxillofac Radiol. 2000 Mar;29(2):70-5. 